\title{
Os desafios enfrentados pela equipe editorial do Journal of Venomous Animals and Toxins including Tropical Diseases na transição entre dois publishers de renome internacional
}

\author{
Challenges faced by the Journal of Venomous Animals and Toxins including \\ Tropical Diseases' editorial team in the transition between two internationally \\ renowned publishers
}

\author{
Claudia Vilalva Cassaro \\ Mestre em Doenças Tropicais \\ Universidade Estadual Paulista \\ claudia.v.cassaro@gmail.com \\ Selma de Lourdes Pires Martins \\ Mestre em Teoria e Pesquisa em Comunicação \\ Universidade Estadual Paulista \\ selma@cevap.unesp.br \\ Lucilene Delazari dos Santos \\ Doutora em Biologia Molecular \\ Universidade Estadual Paulista \\ lucilenebio@gmail.com \\ Benedito Barraviera \\ Doutor em Medicina Interna \\ Universidade Estadual Paulista \\ bbviera@gnosis.com.br
}

\author{
Juliana Siani Simionato \\ Mestre em Teoria e Pesquisa em Comunicação \\ Universidade Estadual Paulista \\ juliana@cevap.unesp.br \\ José Claudio Pires Martins \\ Graduado em Tecnologia em Gestão Ambiental \\ Universidade Estadual Paulista \\ sdsbtu@gmail.com
}

Rui Seabra Ferreira Junior

Universidade Estadual Paulista

Doutor em doenças tropicais rui.seabra@unesp.br

Resumo

The Journal of Venomous Animals and Toxins, criado em 1995 no Centro de Estudos de Venenos e Animais Peçonhentos (CEVAP) da Universidade Estadual Paulista (UNESP), São Paulo, Brasil, foi a primeira revista digital brasileira. Em 1998 foi selecionada para integrar a base SciELO (Scientific Electronic Library Online), e em 2003, para se tornar mais abrangente, as Doenças Tropicais foram incluídas no seu escopo. Denominouse então The Journal of Venomous Animals and Toxins including Tropical Diseases. Em 2006 foi selecionada para integrar duas Bases internacionais estratégicas: a Web of Science da Clarivate Analytics, que publica anualmente o Fator de Impacto, e a Scopus da Elsevier, que publica o Cites per Doc e o CiteScore. Como estes índices se estabilizaram até 2012 entre 0.30 e 0.50, o Conselho Editorial decidiu estabelecer parceria com a BioMed Central Springer-Nature, um publisher comercial de acesso aberto e de renome internacional. Houve, nesta ocasião, uma reestruturação importante do periódico, incluindo a publicação em fluxo contínuo e a indexação no PubMed Central, com vistas à melhoria da qualidade e do aumento das métricas. Em 2018 o Fator de Impacto alcança o valor de 2.935, o Cites per Doc o de 2.973 e o CiteScore de 2.630. Em seis anos os

doi: $10.28998 /$ cirev.2020.7ne.47-54

Esta obra está licenciada sob uma Licença Creative Commons.

Submetido em: 29/02/2020

Aceito em: $12 / 03 / 2020$

Publicado em: 30/03/2020 
Claudia Vilalva Cassaro / Juliana Siani Simionato / Selma de Lourdes Pires Martins / José Claudio Pires Martins / Lucilene Delazari dos Santos / Rui Seabra Ferreira Junior /

Benedito Barraviera

indicadores tiveram um incremento substancial, ou seja, de 0.5 para 2.9. Tendo em vista os elevados investimentos necessários para renovação do contrato de parceria, e por tratar-se de uma publicação acadêmica e não-comercial, o corpo editorial decidiu manter o acesso aberto e tentar a sustentabilidade financeira do periódico a médio prazo, trazendo a publicação de volta ao Brasil a partir de 2019. A equipe SciELO acolheu a corajosa iniciativa e tornou-se o novo parceiro neste desafio. As estratégias adotadas na transição estão descritas em detalhes no "relato de experiência".

\title{
Palavras-chave
}

Periódicos científicos eletrônicos. Publisher. Acesso aberto. Sustentabilidade.

\begin{abstract}
The Journal of Venomous Animals and Toxins, created in 1995 at the Center for the Studies of Venoms and Venomous Animals (CEVAP) at São Paulo State University (UNESP), São Paulo, Brazil, was the first Brazilian digital journal. In 1998 it was selected to be part of the SciELO database (Scientific Electronic Library Online), and in 2003 to become more comprehensive Tropical Diseases were included in its scope. It was then called The Journal of Venomous Animals and Toxins including Tropical Diseases (JVATiTD). In 2006 it was selected to be part of two strategic international databases: Web of Science from Clarivate Analytics, which annually publishes the Impact Factor, and Scopus from Elsevier, which publishes Cites per Doc and CiteScore. As these metrics stabilized until 2012 between 0.30 and 0.50 the Editorial Board decided to partner with BioMed Central Springer-Nature, an internationally renowned open access commercial publisher. There was a major restructuring of the journal at that occasion, including streaming publishing and indexing in PubMed Central, with a view to improving quality and increasing metrics. In 2018 the Impact Factor reaches 2,935, Cites per Doc 2,973 and CiteScore 2,630. In six years, the indexes increased substantially, ie from 0.5 to 2.9. In view of the high investments required to renew the partnership agreement, and because JVATiTD is an academic and non-commercial publication, the editorial board decided to maintain open access and seek the financial sustainability of the journal in the medium term, bringing the publication back to Brazil from 2019. The SciELO team decided to support this brave initiative and became the new partner in this challenge. The strategies adopted in this transition are described in detail in the "experience report".
\end{abstract}

\section{Keywords}

Electronic scientific journals. Publisher. Open access. Financial sustainability.

\section{INTRODUÇÃO}

As revistas ou periódicos científicos são publicações seriadas, fasciculadas, numeradas progressiva e cronologicamente e publicadas em intervalos regulares sem previsão de término. As duas primeiras revistas científicas que se tem notícia surgiram em 1665, com dois meses de diferença entre uma e outra. O periódico francês Journal des Sçavants começou a ser publicado em Paris, no mês de janeiro de 1665, e o Philosophical Transactions da Royal Society of London em março, sendo ambas consideradas os protótipos das revistas científicas mundiais. Durante décadas, suas contribuições foram publicadas em meio impresso, no formato de revistas, livros ou até mesmo folhetos (MEADOWS, 2001). Tais produções são sempre assinadas por um ou mais autores e avaliadas pelos editores e pareceristas escolhidos de acordo com o assunto abordado (FACHIN; HILLESHEIM, 2006; GARRIDO; RODRIGUES, 2010; STUMPF,1998). Elas desempenham papel crucial na divulgação da ciência mundial (STUMPF, 1996; MIRANDA, PEREIRA, 1996).

No Brasil, as primeiras publicações científicas datam do século XIX, e já surgiram com o objetivo de difundir o conhecimento divulgado em outros países. Naquela época os jornais eram cotidianos e não especializados. "O Patriota", de 1813, é considerado o primeiro jornal a tratar de "ciência” (FREITAS, 2006). Já em 1956, o Instituto Brasileiro de Bibliografia e Documentação lança o "Periódicos Brasileiros de Cultura" (STUMPF,1998).

No âmbito das Doenças Tropicais e da Toxinologia necessitamos resgatar e registrar duas revistas brasileiras que participaram efetivamente desta história. Uma das mais antigas é a Memórias do Instituto Oswaldo Cruz, fundada em 1909 pelo próprio cientista Oswaldo Cruz. Foi criada com o objetivo de publicar manuscritos de Parasitologia e Medicina Tropical. 
A outra, que também faz parte desta história, é a Memórias do Instituto Butantan, criada em 1918 e dedicada à publicação de temas relacionados ao Ofidismo, Zoologia Médica, Parasitologia, Bacteriologia, Imunologia, Virologia, Fisiopatologia, Farmacologia e Química Experimental. Durante sua existência, que ocorreu entre 1918 e 1993, foram publicadas obras de renomados pesquisadores, tais como Vital Brazil, Eduardo Vaz, José Lemos Monteiro, Alcides Prado, Flavio da Fonseca, Alphonse Richard Hoge, Alcides Prado, Gastão Rosenfeld, Otto Bier, Jandyra Planet do Amaral, Wolfgang Büecherl, Paulo Vanzolini, entre outros.

Com o aumento significativo tanto na produção da ciência quanto na publicação das revistas, elas se consolidaram como importante canal de informação para cientistas, pesquisadores, professores e alunos. As novas tecnologias ajudaram no aumento e na disseminação dos conteúdos. Um dos maiores desafios da década de 1990 foi: "Como imprimir todas estas informações e enviar aos interessados pelo correio?". Visando contornar o problema, em 1991 foi fundada a Los alamos e-print archives nos Estados Unidos, pioneira em distribuir papers eletrônicos automaticamente.

Em 1994, existiam no mundo cerca de 100 revistas eletrônicas científicas, embora não houvesse nenhuma ainda no Brasil. O CEVAP (Centro de Estudos de Venenos e Animais Peçonhentos da UNESP) participou desta história e lançou a sua revista científica digital em 1995, denominada The Journal of Venomous Animals and Toxins - ISSN 0104-7930.

\section{EVOLUÇÃO DO THE JOURNAL OF VENOMOUS ANIMALS AND TOXINS INCLUDING TROPICAL DISEASES: DA CRIAÇÃO À ÚLTIMA TRANSIÇÃO}

Em 1995, o Centro de Estudos de Venenos e Animais Peçonhentos da UNESP (CEVAP) lançou, no mercado editorial, o The Journal of Venomous Animals and Toxins (JVAT) (BARRAVIERA, 2015), a primeira revista científica digital brasileira. Entre 1995 e 2002, a periodicidade era semestral, sua distribuição era feita por meio de disquetes de 3.5”, e por CD-Roms entre 2000 e 2002. A partir de 2003 adquiriu-se o domínio www.jvat.org.br, momento em que tanto o acesso aos artigos publicados quanto o processo de submissão tornaram-se totalmente digitais (MARTINS et al., 2018). Também em 2003, para torná-la mais abrangente, a temática das Doenças Tropicais foi inserida no escopo da revista, passando a denominar-se The Journal of Venomous Animals and Toxins including Tropical Diseases (JVATiTD) - ISSN 1678-9199. Entre 2005 e 2012 a publicação era trimestral, mantendo o seu Fator de Impacto, que foi conquistado em 2006 junto à Web of Science, estável e em torno de 0.5.

Em 2013, a equipe do JVATiTD estabeleceu parceria com a BioMed Central SpringerNature, um publisher internacional, a fim de ampliar suas indexações, manter o acesso aberto e aumentar o fator de impacto (MARTINS et al., 2018; BARRAVIERA, 2013). Neste mesmo ano foi conquistada a indexação nas bases Pubmed e Pubmed Central (SIMIONATO et al., 2018).

Em 2018, ou seja, seis anos após o início da parceria, houve necessidade de renovação do contrato. Em decorrência dos elevados custos propostos pelos novos administradores da BioMed Central (BMC), os editores - impossibilitados de assumir tal compromisso, haja vista que a publicação é acadêmica e não comercial - optaram por deixar o publisher internacional. Assim, o desligamento foi solicitado e partiu-se em busca de um novo parceiro que pudesse manter o acesso aberto e publicar a revista com a mesma qualidade conquistada. Além disso, tendo em vista a escassez crescente de recursos financeiros públicos para financiar a publicação, pensou-se numa alternativa de sustentabilidade financeira a médio prazo, cobrando-se o suficiente por artigo publicado (article processing charge - APC).

A Scientific Electronic Library Online (SciELO) é uma biblioteca digital de acesso aberto (PACKER, 1998), e o JVATiTD faz parte da coleção desde 1998. O SciELO acolheu e aceitou dois importantes desafios, a saber: continuar publicando a revista em fluxo contínuo e também atuar, a partir de 2019, como publisher. 
Claudia Vilalva Cassaro / Juliana Siani Simionato / Selma de Lourdes Pires Martins / José Claudio Pires Martins / Lucilene Delazari dos Santos / Rui Seabra Ferreira Junior /

Benedito Barraviera

A seguir, elencamos os principais desafios enfrentados pela equipe do JVATiTD e do SciELO nesta transição:

- Desenvolvimento de um website provisório utilizando-se o antigo domínio registrado pela BioMed Central (www.jvat.org): Além da homepage do JVATiTD já existente no SciELO < http://www.scielo.br/scielo.php?script=sci serial\&pid=1678-9199\&lng=en\&nrm= iso $>$ (Figura 1), optou-se por criar um website provisório e independente, mais abrangente e customizável para adequar-se ao dinamismo do periódico (Figura 2). Tal projeto foi finalizado, com sucesso, no início de 2019. Possui links para a submissão e gestão de manuscritos, que é realizada por meio do Scholar One Manuscripts https://mc04.manuscriptcentral.com/jvatitd-scielo - um moderno sistema de fluxo editorial sob responsabilidade do SciELO, além de outras funcionalidades.

Figura 1. Homepage do Journal of Venomous Animals and Toxins including Tropical Diseases na biblioteca SciELO.

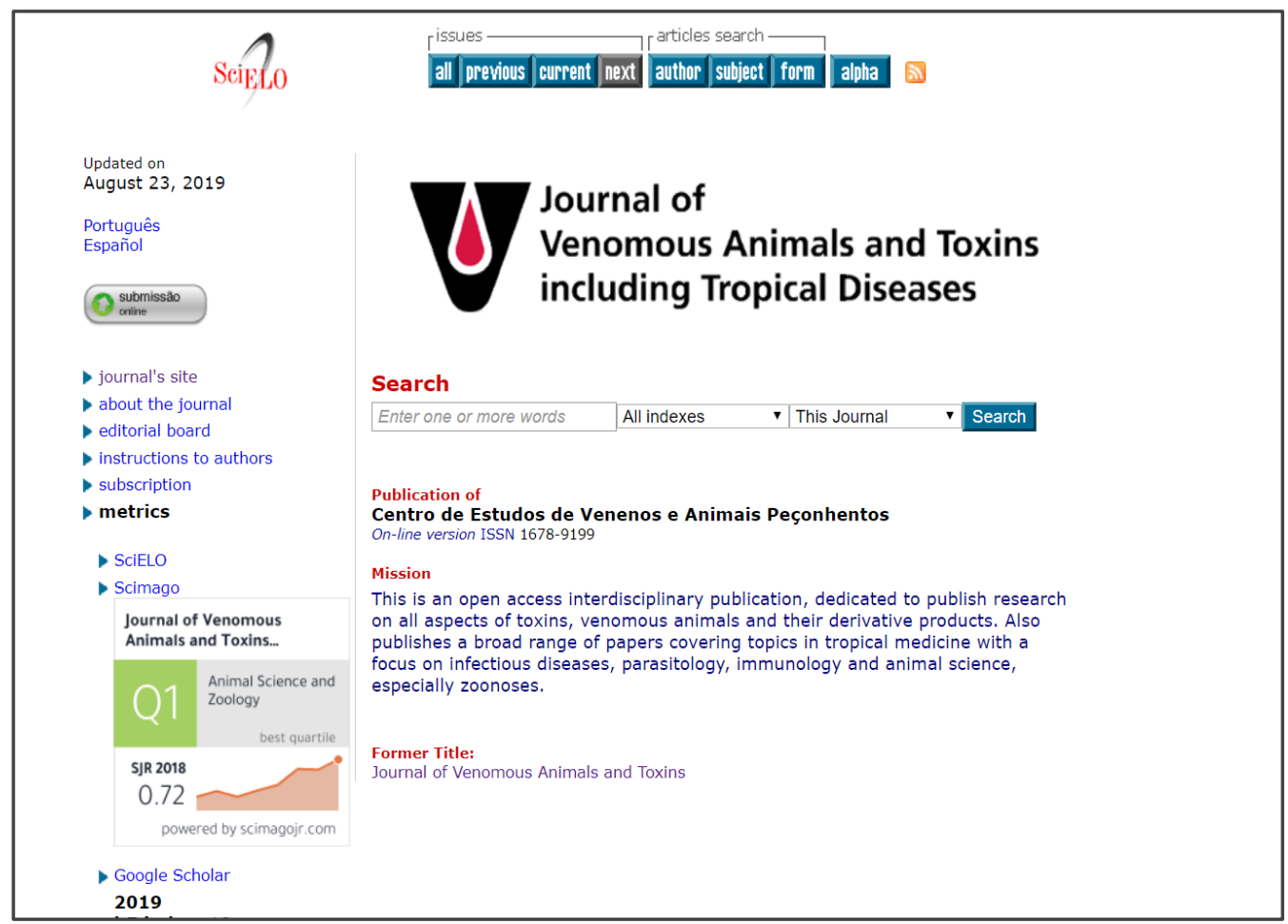


Figura 2. Homepage do Journal of Venomous Animals and Toxins including Tropical Diseases no domínio

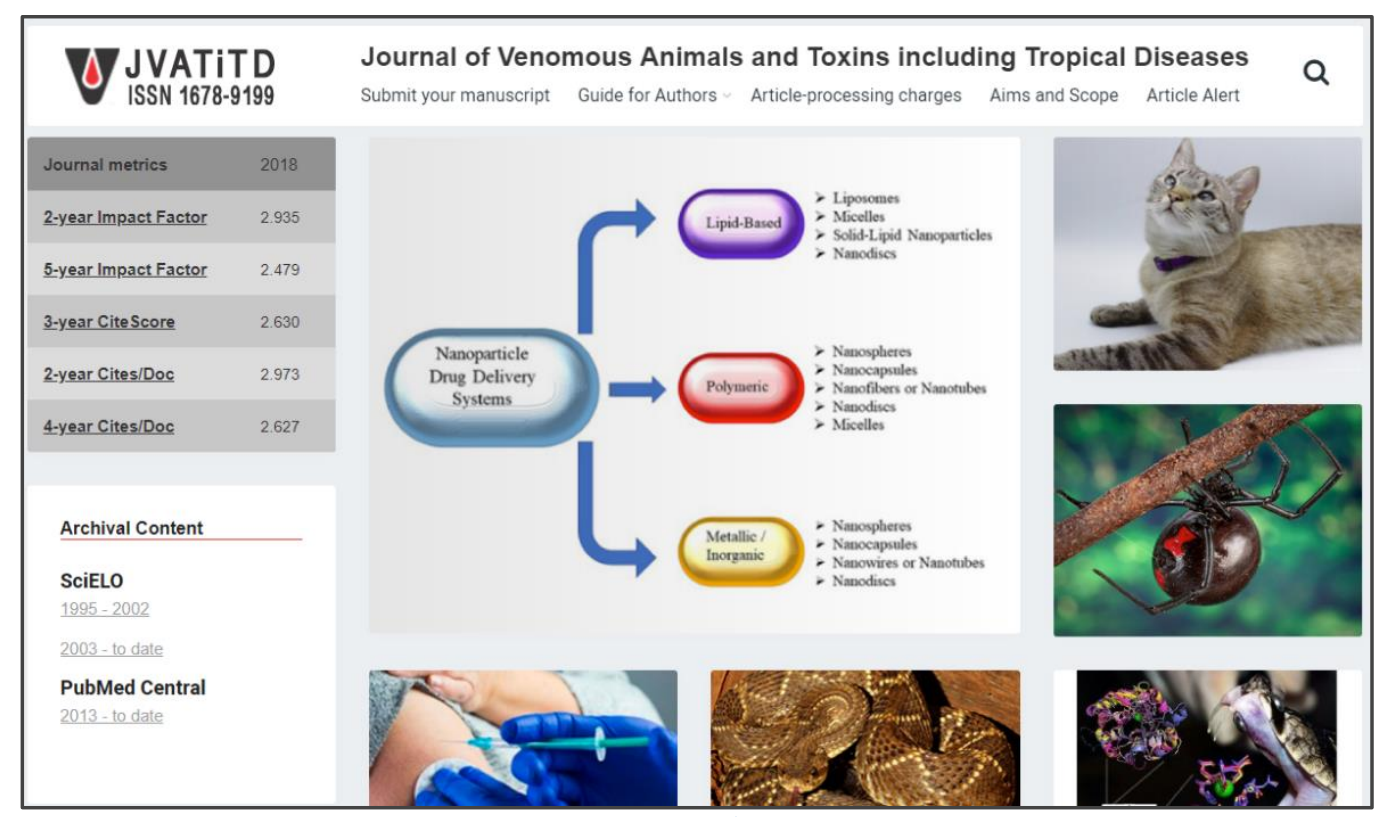

Fonte: www.jvat.org

- Transferência do domínio do site e redirecionamento de domínios: Até o momento da transição, o site do JVATiTD estava vinculado à Biomed Central, que gentilmente cedeu e auxiliou na transferência do domínio www.jvat.org para o Brasil. Uma página referente à revista ainda está disponível na BioMed Central (Figura 3), a qual possui, sob arquivamento, todos os artigos publicados entre 2013 e 2018. Aproveitou-se o momento para reutilizar e redirecionar os navegadores para os domínios brasileiros já existentes, ou seja, as páginas www.jvat.org.br e www.jvat.com.br, a fim de facilitar o acesso dos autores e pesquisadores à nova homepage.

Figura 3. Homepage do Journal of Venomous Animals and Toxins including Tropical Diseases mantido na BioMed Central, com acesso a todos os artigos publicados no período da parceria.

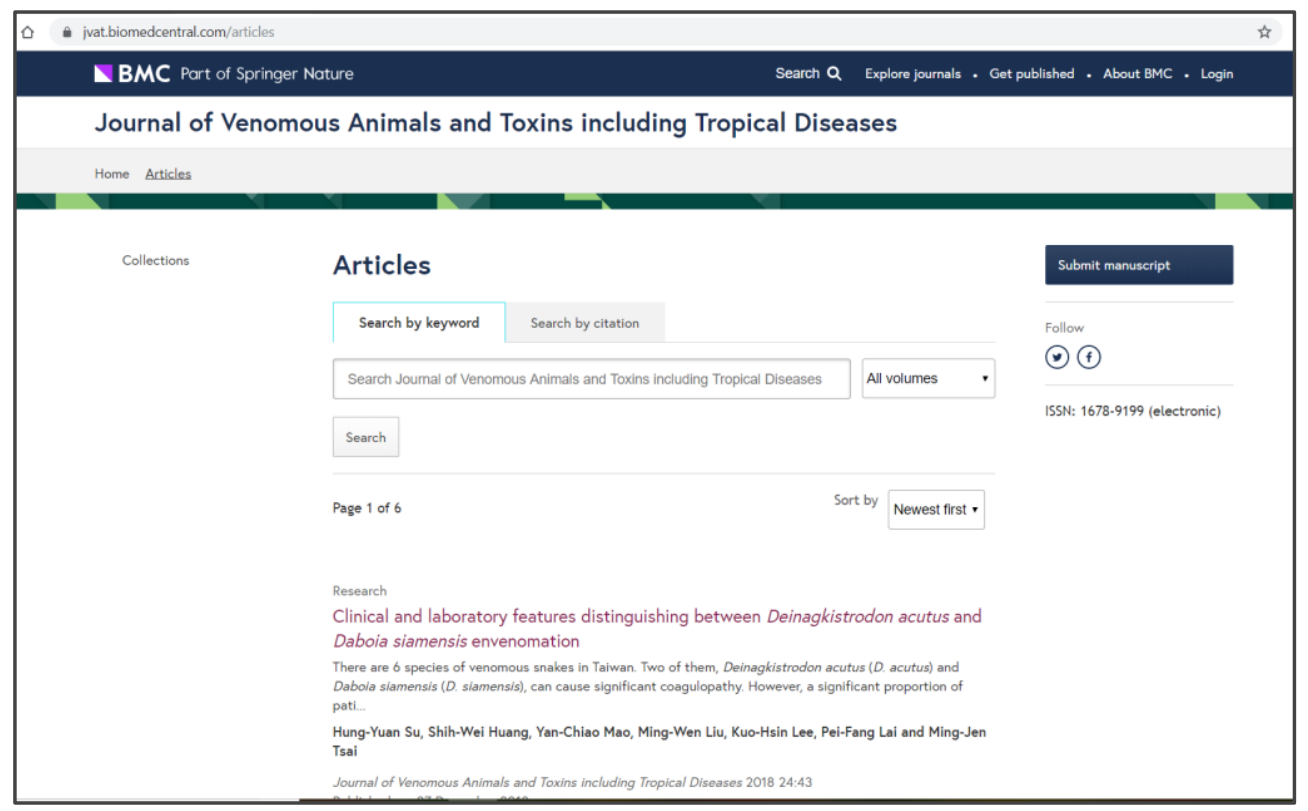


- Troca do DOI number: O Digital Object Identifier (DOI) number é reconhecidamente um importante critério para identificação de arquivos científicos digitais. Dessa forma, foi necessário a tramitação e um acordo entre a BMC e o SciELO para este último ser o novo DOI provider.

- Capacitação da equipe para a utilização do novo sistema editorial: Por ocasião da transição de publisher houve o primeiro contato da equipe editorial do JVATiTD com o sistema Scholar One Manuscripts. Entretanto, todas as dificuldades foram contornadas pela capacitação oferecida pelo SciELO, bem como o suporte constante da equipe de gestão editorial do novo publisher.

- Definição de novos Guidelines para a submissão de manuscritos: Anteriormente, o JVATiTD seguia os guidelines definidos pela BioMed Central. Com a nova parceria, o corpo editorial debruçou-se sobre as necessidades e formatos requeridos pela revista, delineando novas normas que atendessem ao formato da publicação, adaptados a partir do padrão mundial vigente.

Deve ser salientado que o JVATiTD originalmente era um periódico acadêmico, não comercial, que sobrevivia com recursos públicos provindos ora da Universidade, ora das agências de fomento brasileiras. A partir de 2013, em decorrência da parceria estabelecida entre a Universidade Estadual Paulista (UNESP) e a BMC, tornou-se um periódico considerado comercial, cobrando-se um Article Processing Charge (APC) para publicação, embora o Editor-chefe, bem como todos os Editores associados, não fossem remunerados.

A fim de priorizar a missão do acesso aberto à ciência, optou-se por reafirmar que o JVATiTD é uma publicação acadêmica, não comercial, ou seja, sem fins lucrativos. As taxas de submissão (article processing charge - APC) foram revistas e diminuídas para 500 dólares, para autores estrangeiros, e 300 dólares para os nacionais. Tal decisão é uma clara ação de valorização e de incentivo à produção científica nacional, haja vista que hoje, com o crescimento das métricas, o JVATiTD tem excelente competitividade internacional. Além disso, a decisão deverá estimular os autores a escolher o JVATiTD para publicar e otimizar recursos financeiros. E, em decorrência da escassez de recursos públicos para editoração, esta modesta captação poderá, a médio prazo, tornar o periódico sustentável financeiramente.

As ações adotadas foram satisfatórias, estão em processo de avaliação e hoje todos os percalços da transição foram superados. Destacamos que nenhuma das indexações do JVATiTD foi perdida, em especial a do Pubmed Central (Figura 4), que garante prestígio e enorme visibilidade.

Figura 4. Indexação do Journal of Venomous Animals and Toxins including Tropical Diseases no PubMed Central.

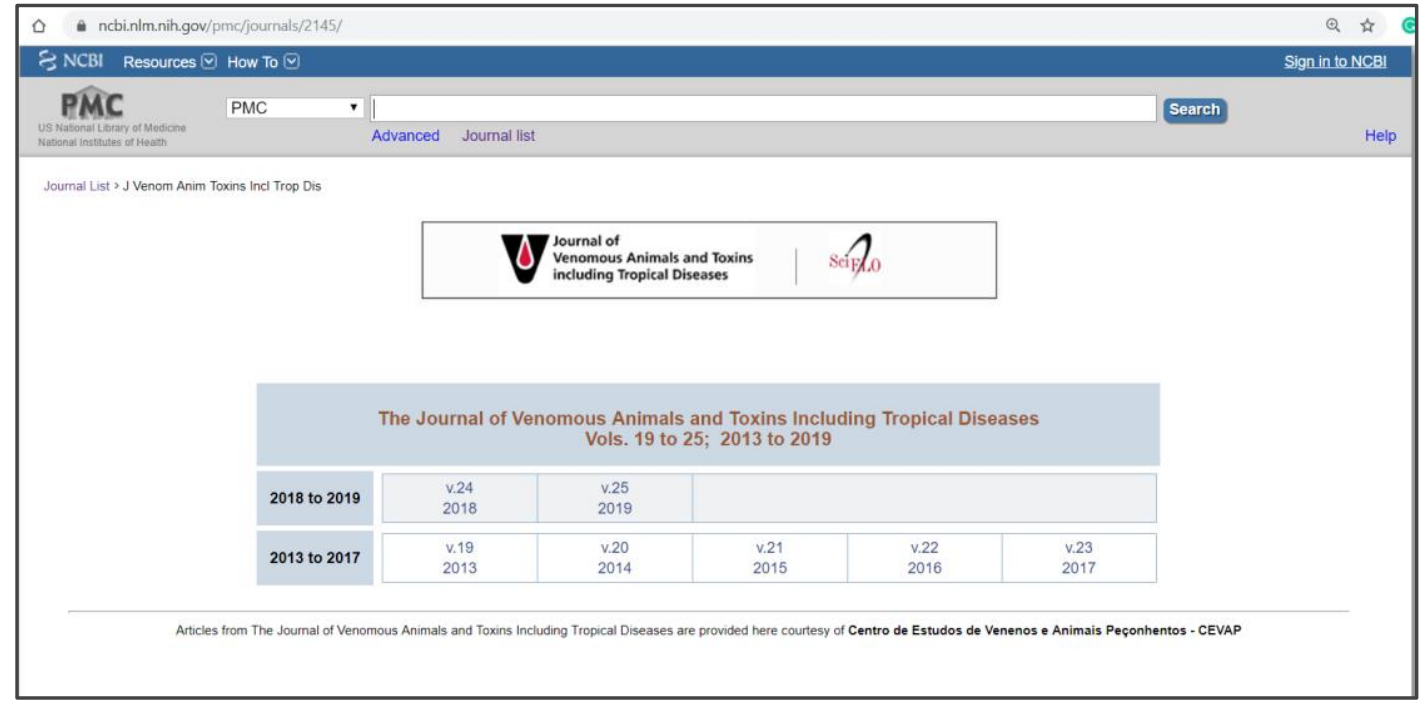

Por meio de ações inovadoras, os indicadores bibliométricos do JVATiTD vêm apresentando importante crescimento, atingindo em 2018 o Fator de Impacto (Journal Citation 
Reports) de 2.935, o CiteScore (Scopus), de 2.630, e o Cites per Doc (Scimago Journal \& Country Rank), de 2.973 (Figura 5).

Figura 5. Crescimento das métricas do Journal of Venomous Animals and Toxins including Tropical Diseases

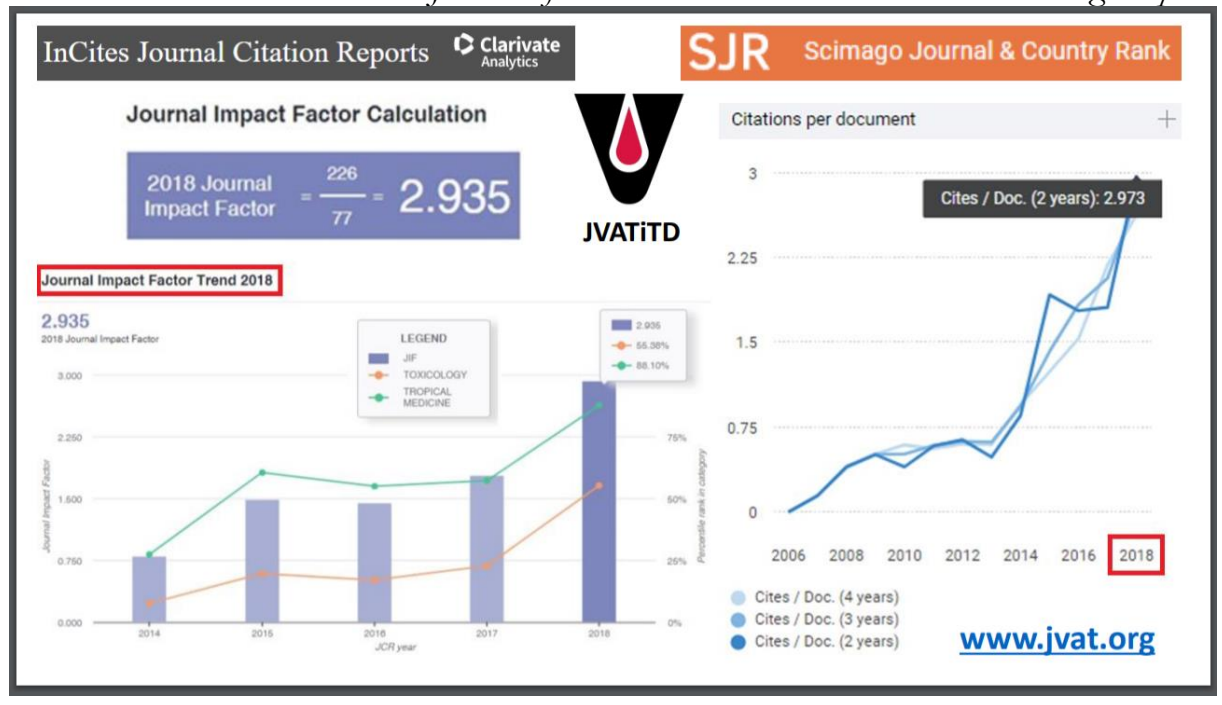

\section{CONSIDERAÇÕES FINAIS}

A transição de um publisher internacional para um nacional mostra o risco, a coragem, a ousadia e o pioneirismo com que a equipe editorial do JVATiTD enfrenta os atuais desafios impostos pelo mundo globalizado. A parceria de seis anos com a BioMed Central Springer-Nature proporcionou grande aprendizado, em especial na profissionalização da editoração do periódico. Houve incremento no prestígio, na produtividade, na visibilidade e foi imprescindível para o significativo aumento do fator de impacto e na conquista das demais métricas.

Devido aos elevados custos da publicação e em busca de sustentabilidade financeira futura, uma nova parceria com o SciELO foi estabelecida em 2019. Esperamos que a visibilidade e o crescimento dos indicadores bibliométricos continuem, embora saibamos o grande desafio que enfrentaremos em 2020. Apesar disso, a equipe editorial tenta se reinventar com dinamismo, criatividade e inovação, fenômeno que já ocorre há décadas quando o JVATiTD foi criado em 1995 no formato digital, época em que ainda não havia internet comercial no Brasil.

\section{AGRADECIMENTOS}

A equipe editorial do JVATiTD agradece à BioMed Central Springer-Nature pelos profícuos anos de parceria, bem como pelo profissionalismo e solicitude em todo o processo de transição. Nossos agradecimentos especiais à Dana Berry, Linda van der Wende, Laurence Cheeseman e Mariana Biojone Brandão. Agradece, também, a toda equipe de gestão editorial do SciELO e colaboradores pela capacitação e suporte, em especial ao Alex Mendonça, Carolina Tanigushi, Luis Gustavo S. Gomes, Daniel Buganza, Francine Curivil, Rondineli Gama Saad, Solange Santos e Abel Packer. O editor-chefe faz um agradecimento especial à equipe da Visual Informática, em especial ao Silvio Teixeira, Luiz Primati e Natasha Primati, além do José Cláudio Pires Martins, Milton Shintaku e à própria Claudia Vilalva Cassaro, autora deste relato, os quais não mediram esforços para tornar esta proposta viável. 
Claudia Vilalva Cassaro / Juliana Siani Simionato / Selma de Lourdes Pires Martins / José Claudio Pires Martins / Lucilene Delazari dos Santos / Rui Seabra Ferreira Junior /

Benedito Barraviera

\section{REFERÊNCIAS}

BARRAVIERA, B. CEVAP journal towards a new phase. Journal Of Venomous Animals And Toxins Including Tropical Diseases, [s.l.], v. 19, n. 1, 2013.

BARRAVIERA, B. CEVAP Journal: the first Brazilian electronic scientific publication turns 20 years old. Journal Of Venomous Animals And Toxins Including Tropical Diseases, [s.l.],v. 21, n. 1, dez. 2015.

FACHIN, G. R. B.; HILLESHEIM, A. I. de A., Periódico Científico: padronização e organização. Florianópolis: Ed. da UFSC, 2006.

FREITAS, M. H. Considerações acerca dos primeiros periódicos científicos brasileiros. Ciência da Informação, v. 35, n. 3, p. 54-66, set/dez 2016.

GARRIDO, I. dos S.; RODRIGUES, Rosangela Schwarz. Portais de periódicos científicos online: organização institucional das publicações. Perspectivas em Ciência da Informação, v.15, n.2, p.56-72, mai/ago. 2010.

MARTINS, S. de L. P. et al. Do disquete às nuvens: A saga da primeira revista eletrônica científica brasileira. Ciência da Informação em Revista. v. 5, n.esp., p. 86-100, 2018.

MEADOWS, J. Os periódicos científicos e a transição do meio impresso

para o eletrônico. Revista de Biblioteconomia de Brasília, v. 25, n.1, p. 5-14, 2001.

MIRANDA, D. B.; PEREIRA, M. N. de F. O periódico científico como veículo de comunicação: uma revisão de literatura. Ciência da Informação, v. 25, n. 3, 1998.

NAZARÉ, E. A. et al. O digital object identifier (DOI) em periódicos científicos eletrônicos de comunicação e informação. RDBCI: Revista Digital Biblioteconomia e Ciência da Informação, v. 15, n. 3, p. 533-549, set/dez 2017.

PACKER, A. L. et al. SciELO: uma metodologia para publicação eletrônica. Ciência da Informação, v. 27, n. 2, 1998.

SIMIONATO, J. S. et al. Como aumentar o fator de impacto nas bases Web of Science (WoS) e Scopus (Scimago): Ações implementadas pelo Journal of Venomous Animals and Toxins including Tropical Diseases. Ciência da Informação em Revista. v. 5, n. esp., p. 58-67, 2018.

STUMPF, I. R. C. Passado e futuro das revistas científicas. Ciência da Informação, v. 25, n. 3, 1996.

STUMPF, I. R. C. Reflexões sobre as revistas brasileiras. Intertexto, v. 1, n.3, p. 1-10, 1998. 